\title{
CHARACTERIZATIONS AND GENERALIZATIONS OF CONTINUITY
}

\author{
J. M. ASH, J. COHEN, C. FREILING, L. GLUCK, E. RIEDERS, AND G. WANG
}

(Communicated by Andrew Bruckner)

\begin{abstract}
The condition $f(x+2 h)-2 f(x+h)+f(x)=o(1)$ (as $h \rightarrow 0)$ at each $x$ is equivalent to continuity for measurable functions. But there is a discontinuous function satisfying $2 f(x+2 h)-f(x+h)-f(x)=o(1)$ at each $x$. The question of which generalized Riemann derivatives of order 0 characterize continuity is studied. In particular, a measurable function satisfying $\sum_{i=1}^{n} \alpha_{i} f\left(x+\beta_{i} h\right) \equiv 0$ must be a polynomial. On the other hand, for any Riemann derivative of order 0 and any $p \in[1, \infty]$, generalized $L^{p}$ continuity is equivalent to $L^{p}$ continuity almost everywhere.
\end{abstract}

\section{INTRODUCTION}

In Zygmund's book Trigonometric series, the space of Lipschitz 1 functions is defined by the relation $|g(x+h)-g(x)|=O(h)$, and what is now called the Zygmund space $\Lambda_{*}$ is defined by the relation $\mid g(x+2 h)-2 g(x+h)+$ $g(x) \mid=O(h)$. These spaces are different. However, Zygmund points out that if $0<\alpha<1$, the conditions

$$
|g(x+h)-g(x)|=O\left(h^{\alpha}\right)
$$

and

$$
|g(x+2 h)-2 g(x+h)+g(x)|=O\left(h^{\alpha}\right)
$$

are equivalent $[\mathrm{Zy}, \mathrm{p} .44]$. This suggests that for measurable functions the condition

$$
f(x+2 h)-2 f(x+h)+f(x)=o(1) \quad(\text { as } h \rightarrow 0)
$$

might be equivalent to continuity of $f$. Considering, at the point $x=0$, the example

$$
\begin{array}{ll}
f(h)=n h & \text { if } h=\frac{2 n+1}{2^{j}} \quad(j=0,1, \ldots, n=1,2, \ldots) \\
f(x)=0 & \text { elsewhere }
\end{array}
$$

Received by the editors October 16, 1992.

1991 Mathematics Subject Classification. Primary 26A15, 26A21, 39A10; Secondary 26A24, $30 \mathrm{C} 15$.

Key words and phrases. Generalized continuity, generalized Riemann derivative of order zero.

The work of the first, second, and sixth authors was partially supported by a grant from the Faculty Research and Development Program of the College of Liberal Arts and Sciences, DePaul University. 
shows that this equivalence cannot be true at a single point. This leads to the following definition. Let $\alpha \beta$ be a point of $\mathbb{R}^{n} \times \mathbb{R}^{n}$ with $\sum \alpha_{i}=0$. Let $C_{\alpha \beta}:=\left\{f: \mathbb{R} \rightarrow \mathbb{R}:\right.$ for all $\left.x, \lim _{h \rightarrow 0} \sum_{i=1}^{n} \alpha_{i} f\left(x+\beta_{i} h\right)=0\right\}$. If $f \in C_{\alpha \beta}$, say that $f$ is generalized n-point Riemann differentiable of order 0 with respect to $\alpha \beta$, or more simply, say that $f$ is generalized continuous with respect to $\alpha \beta$.

Remarkably, in view of the above example, it turns out that $f$ is continuous if and only if, for all $x, f(x+2 h)-2 f(x+h)+f(x)=o(1)$. In other words, $C_{(1,-2,1)(2,1,0)}=C$, the set of continuous functions on $\mathbb{R}$. On the other hand, $f(x)=\frac{1}{x} \chi_{\{x \neq 0\}}$ at every $x$ satisfies $\lim _{h \rightarrow 0} 2 f(x+2 h)-f(x+h)-f(x)=0$ so that $C_{(2,-1,-1)(2,1,0)} \supsetneq C$. These two results are special cases of Theorem 1 , which classifies generalized 3-point Riemann derivatives of order 0 into those characterizing continuity (i.e., $C_{\alpha \beta}=C$ ) and those extending continuity (i.e., $C_{\alpha \beta} \supsetneq C$ ).

If, however, one ignores sets of measure zero, then any Riemann derivative of order 0 based on any number of points is a characterizer, rather than an extender, of continuity. This is the content of Theorem 2 . Theorem 3 is an $L^{p}$ version of Theorem 2 .

Now consider generalized $(n+2)$-point Riemann derivatives of order 0 of the form

$$
\lim _{h \rightarrow 0} \sum_{i=0}^{n} a_{i} f\left(x+b^{i} h\right)-\left(\sum_{i=0}^{n} a_{i}\right) f(x)=0 .
$$

To such a difference we associate a polynomial which we call the characteristic polynomial. A simple reduction allows us to assume that $|b|>1$. The characteristic polynomial is $\sum_{i=0}^{n} a_{i} z^{i}$. Theorem 4 shows that such derivatives characterize continuity exactly when all $n$ roots of the characteristic polynomial lie outside of the closed unit disk.

It remains only to classify the general $(n+2)$-point Riemann derivatives of order 0 . By simple change of variable we may assume these have the form

$$
\lim _{h \rightarrow 0} \sum_{i=0}^{n} a_{i} f\left(x+b_{i} h\right)-\left(\sum_{i=0}^{n} a_{i}\right) f(x)=0,
$$

where $b_{0}=1$ and the $\left|b_{i}\right|$ are nondecreasing. Let $b:=b_{1}$ (but if $b_{1}=-1$ let $\left.b:=-\left|b_{2}\right|\right)$. Then for all $i$ let $p_{i}:=\log b_{i} / \log b$, where $\log \beta:=\log |\beta|+i \pi$ when $\beta<0$, so that our derivative can be written as

$$
\lim _{h \rightarrow 0} \sum_{i=0}^{n} a_{i} f\left(x+b^{p_{i}} h\right)-\left(\sum_{i=0}^{n} a_{i}\right) f(x)=0,
$$

where a case-by-case check shows that $p_{0}=0$ and $\Re p_{i}>0$ when $i \geq 1$. By the now obvious analogy with the situation described in the previous paragraph, we are led to the following conjecture.

Conjecture. If for all $x$ we have

$$
\lim _{h \rightarrow 0} \sum_{i=0}^{n} a_{i} f\left(x+b^{p_{i}} h\right)-\left(\sum_{i=0}^{n} a_{i}\right) f(x)=0,
$$


where $p_{0}=0$ and $\Re p_{i}>0$ when $i \geq 1$, then $f$ is continuous if all the roots of the quasipolynomial $\sum_{i=0}^{n} a_{i} z^{p_{i}}$ have moduli strictly greater than 1 .

Theorem 5 shows that the converse of the conjecture is true. It follows that a positive resolution of the conjecture amounts to a complete classification-to decide whether an $(n+2)$-point Riemann derivative of order 0 characterizes continuity one would need only check whether its characteristic quasipolynomial has any zeros in the unit disk. Theorem 6 confirms the special case of the conjecture that arises when $\left|a_{0}\right|>\sum_{i=1}^{n}\left|a_{i}\right|$ and all $b^{p_{i}}$ are positive. Theorem 7 points out that quasipolynomials often have a finite number of roots. This feature provides a little more evidence in support of the conjecture.

Theorem 8 is of a slightly different flavor. Suppose we know only that $f$ is measurable and that, for every $x$ and every $h, f$ satisfies the difference equation

$$
\sum_{i=0}^{n} a_{i} f\left(x+b_{i} h\right)=0 \text {. }
$$

Then $f$ must be a polynomial and the degree of $f$ is controlled by the behavior of the sums $\sum a_{i} b_{i}^{j}$.

\section{RESUlts}

Suppose that a measurable real-valued function satisfies

$$
\lim _{h \rightarrow 0} \alpha_{1} f\left(x+\beta_{1} h\right)+\alpha_{2} f\left(x+\beta_{2} h\right)+\alpha_{3} f\left(x+\beta_{3} h\right)=0, \quad \text { all } x,
$$

where $\sum \alpha_{i}=0, \prod \alpha_{i} \neq 0$, and the $\beta_{i}$ are distinct, with $\left|\beta_{1}\right| \geq\left|\beta_{2}\right| \geq\left|\beta_{3}\right|$. Condition (1) holds for continuous functions; the converse, however, is not always true.

Theorem 1. A function satisfying (1) everywhere is continuous whenever

(i) $\beta_{3}=0$ and either of the following two conditions holds.

(ii) $\left|\beta_{1}\right|>\left|\beta_{2}\right|$ and $\left|\alpha_{2}\right|>\left|\alpha_{1}\right|$,

(iii) $\beta_{1}=-\beta_{2}$ and $\alpha_{1} \neq \alpha_{2}$.

If either (i) fails or both (ii) and (iii) fail, then there is a discontinuous function satisfying (1).

Proof. We begin with a full set of counterexamples. If $\beta_{3} \neq 0$, then the characteristic function of $\{0\}$ is discontinuous and satisfies (1). Henceforth, we will take $\beta_{3}=0$.

Now let $a:=\alpha_{2} / \alpha_{1}$ and $b:=\beta_{1} / \beta_{2}$, and replace $h$ by $h / \beta_{2}$ so that condition (1) becomes

$$
\lim _{h \rightarrow 0}[f(x+b h)+a f(x+h)-(1+a) f(x)]=0, \quad \text { all } x,
$$

and (ii) and (iii) become

(iii) $|b|>1$ and $|a|>1$,

(iii') $b=-1$ and $a \neq 1$.

Notice that $a \neq-1$ since $a=-1$ corresponds to $\alpha_{3}=0$. Also, $b \neq 1$ since the $\beta_{i}$ are distinct.

If $|b|>1$ and $|a| \leq 1$, let $F(x):=|x|^{\log _{|b|}(-a)}$ if $x \neq 0$ and let $F(0)=0$. More explicitly, when $x \neq 0$,

$$
F(x)=|x|^{\log _{|b|}(|a|)}\left\{\cos \left(\left(\log _{|b|}|x|\right)(\arg (a)+\pi)\right)+i \sin \left(\left(\log _{|b|}|x|\right)(\arg (a)+\pi)\right)\right\} .
$$


Note that the function $F$ satisfies (2) but is discontinuous at 0 . To get a real-valued counterexample, take, for example,the real part of $F$.

Also, if $b=-1$ and $a=1$, the function $\operatorname{sgn}(x)$ satisfies (2) but is discontinuous at 0 .

It now remains to prove the positive results, namely, that condition (2) together with either $\left(\mathrm{ii}^{\prime}\right)$ or (iii') implies the continuity of $f$. The latter is easy, since

$$
\begin{aligned}
f(x+h)-f(x)= & \frac{-a}{1-a^{2}}\{f(x-h)+a f(x+h)-(1+a) f(x)\} \\
& +\frac{1}{1-a^{2}}\{f(x+h)+a f(x-h)-(1+a) f(x)\}
\end{aligned}
$$

and as $h \rightarrow 0$ the first quantity in curly brackets tends to zero by hypothesis. The substitution of $-h$ for $h$ shows that the second quantity does also. Next, observe that if condition (ii') holds, then the identity

$$
\begin{aligned}
f(x+ & \left.b^{2} h\right)-a^{2} f(x+h)+\left(a^{2}-1\right) f(x) \\
= & {[f(x+b(b h))+a f(x+b h)-(1+a) f(x)] } \\
& -a[f(x+b h)+a f(x+h)-(1+a) f(x)]
\end{aligned}
$$

allows us to assume without loss of generality that $b>1$, and the coefficient of $f(x+h)$ is $-a$, where $a>1$.

In short, we may now suppose that $b>1, a>1$, and

$$
\lim _{h \rightarrow 0}(f(x+b h)-a f(x+h)+(a-1) f(x))=0 \text { for all } x .
$$

Theorem 1 will be proved if we can conclude that $f$ is continuous.

Let $E=\{x: f$ is discontinuous at $x\}$. We will successively show that $E$ has the following properties:

(A) It has measure 0 .

(B) It is perfect, i.e., closed and without isolated points.

(C) If it is nonempty, then it is dense in some nontrivial interval.

Thus, if $E$ is nonempty, because of $(C)$ it is dense in some interval, but because it is closed (see (B)), it must contain that interval. This contradicts (A), which proves Theorem 1 .

Proof of (A). This is a special case of the first of the following results.

Theorem 2. If $f$ is real valued and measurable and if $\sum_{i=1}^{n} \alpha_{i} f\left(x+\beta_{i} h\right)=o(1)$ as $h \rightarrow 0$ for all $x \in A$, where $\sum \alpha_{i}=0$, then $f$ is continuous at almost every point of $A$.

Theorem 3. Let $1 \leq p<\infty$, and let $f$ be measurable and real valued. If

$$
\frac{1}{h} \int_{0}^{h}\left|\sum_{i=1}^{n} \alpha_{i} f\left(x+\beta_{i} t\right)\right|^{p} d t=o(1)
$$

as $h \rightarrow 0$ for all $x \in A$, where $\sum \alpha_{i}=0$, then

$$
\lim _{h \rightarrow 0} \frac{1}{h} \int_{0}^{h}|f(x+t)-f(x)|^{p} d t=0 \quad \text { for almost every } x \in A \text {. }
$$


These theorems can be thought of as assuming the existence of generalized Riemann derivatives of order 0 and concluding the existence of Peano derivatives of order 0 . Their proofs, but not their statements, are contained in [As1]. The theorems in [As1] are asserted for derivatives of order $\geq 1$, but, as was remarked to Ash by Patrick O'Conner in 1969, the proofs in [As1] are, without modification, proofs of Theorems 2 and 3.

Proof of (B). We need the following lemma.

Lemma 1. If $\lim _{h \rightarrow 0} \sum_{i=0}^{n} a_{i}\left(f\left(x+b_{i} h\right)-f(x)\right)=0,\left|a_{0}\right|>\sum_{i=1}^{n}\left|a_{i}\right|$, and $0<\left|b_{0}\right| \leq\left|b_{1}\right| \leq \cdots \leq\left|b_{n}\right|$, then either $f$ is continuous at $x$ or there is $\eta_{x}>0$ such that

$$
\sup _{|h| \in[\eta, b \eta]}|f(x+h)|=\infty
$$

for all $0<\eta<\eta_{x}$, where $b:=\left|b_{n}\right|$.

Furthermore, if all $b_{i}$ are positive, then either $\sup _{h \in[\eta, b \eta]}|f(x+h)|=\infty$ for all $0<\eta<\eta_{x}$ or $f$ is right continuous at $x$. An analogous result holds for left continuity.

Proof. We may assume $x=0, f(0)=0, a_{0}=-1$, and $b_{0}=1$ without loss of generality. Suppose $f$ is not continuous at 0 . There is a sequence $x_{n}$ converging to 0 such that $\left|f\left(x_{n}\right)\right|>c$ for some positive $c$. Choose $0<\beta<$ $1-\sum_{i=1}^{n}\left|a_{i}\right|$. Then there exists $\eta_{0}>0$ such that for $|h|<\eta_{0}$

$$
\left|\sum_{i=1}^{n} a_{i} f\left(b_{i} h\right)-f(h)\right|<\beta c \text {. }
$$

Claim. If $|f(h)|>c$ and $|h|<\eta_{0}$, then $\left|f\left(b_{i} h\right)\right|>a|f(h)|$ for some $i=$ $1, \ldots, n$, where $a:=(1-\beta) / \sum\left|a_{i}\right|>1$.

To see this, simply observe that if otherwise,

$$
|f(h)|<\left|\sum_{i=1}^{n} a_{i} f\left(b_{i} h\right)\right|+\beta c \leq\left(a \sum_{i=1}^{n}\left|a_{i}\right|+\beta\right)|f(h)|=|f(h)|,
$$

a contradiction.

Now given $0<\eta<\eta_{0}$, for any $M>0$, choose $k$ so large that $a^{k} c>M$. Then choose $x_{n_{k}}$ in the sequence such that $\left|b^{k} x_{n_{k}}\right|<\eta$. Because $\left|b_{i}\right| \geq 1$ for each $i$, repeatedly using the claim, we can find elements $c_{j} \in\left\{b_{1}, \ldots, b_{n}\right\}$, $j=1,2, \ldots$, so that for some integer $J=J(k) \geq k+1$,

$$
\left|c_{1} c_{2} \cdots c_{J-1} x_{n_{k}}\right| \leq \eta<\left|c_{1} \cdots c_{J} x_{n_{k}}\right| \text { and }\left|f\left(c_{1} \cdots c_{J} x_{n_{k}}\right)\right| \geq a^{J} c>M \text {. }
$$

(A fine point: it is conceivable that $b_{1}=-1$ and that all but a finite number of the $c_{j}$ 's are equal to $b_{1}$ so that $\left|c_{1} \cdots c_{J} x_{n_{k}}\right|$ never exceeds $\eta$. This does not occur because the claim disallows two consecutive choices of -1 . In fact, from $c_{j+1}=c_{j+2}=-1$ there would follow the absurdity $\left|f\left(c_{1} \cdots c_{j}(-1)^{2} x_{n_{k}}\right)\right|>$ $a^{2}\left|f\left(c_{1} \cdots c_{j} x_{n_{k}}\right)\right|$. In particular, if $n=-b_{1}=1$, continuity of $f$ at 0 is forced.) But $\left|c_{1} \cdots c_{J} x_{n_{k}}\right| \in(\eta, b \eta]$ so that we have $\sup _{|h| \in[\eta, b \eta]}|f(h)|>M$ for any $M$. This proves the first part of the lemma since $M$ may be arbitrarily large.

The other two parts of the lemma have similar proofs. 
We return to the proof of (B). If $f$ satisfies (3) but $x$ is not a point of continuity, then it follows from Lemma 1 that

$$
\limsup _{y \rightarrow x}|f(y)|=\infty \text {. }
$$

Conversely this condition clearly forces $f$ to be discontinuous at $x$, so we may characterize $\mathrm{E}$ by $E=\left\{x: \limsup _{y \rightarrow x}|f(y)|=\infty\right\}$. Now let $a$ be a limit point of $E$. Then there are points of $E$ arbitrarily close to $a$, but each point of $E$ has points where $|f|$ is arbitrarily large arbitrarily close to it, whence $\limsup _{x \rightarrow a}|f(x)|=\infty$ and $a$ is in $E$. This proves that $E$ is closed.

Now suppose that $x$ is an isolated point of $E$. Then $f$ must be either right or left discontinuous at $x$. If, say, $f$ is right discontinuous at $x$, then from Lemma 1 we have that, for every sufficiently small $\eta>0, \sup \{|f(y)|: y \in$ $[x+\eta, x+b \eta]\}=\infty$, which in turn implies that $f$ has a discontinuity in $[x+\eta, x+b \eta]$. This is a contradiction, since $\eta$ can be taken arbitrarily small. Property (B) is established.

Proof of $(\mathrm{C})$. Now suppose that $E$ is nonempty and nowhere dense. By virtue of (B), we may assume that $E$ is perfect. Let $G=E^{c}$, and let $I_{0}$ be a component of $G$. Then $I_{0}$ must have an end point $x_{0}$ in $E$; suppose it is the left endpoint. Since $E$ is perfect, $E$ has a second point to the left of $x_{0}$, so $G$ (being dense) has a finite component, say $\left(x_{1}, y_{1}\right)$. Again $x_{1} \in E$ so that there are points of $E$ below, but arbitrarily close to, $x_{1}$ and consequently components of $G$ below, but arbitrarily close to, $x_{1}$. Let $\left(x_{2}, y_{2}\right)$ be such a component with $b\left(x_{1}-x_{2}\right)<\left(y_{1}-x_{1}\right)$. Having picked the component of $G$ called $\left(x_{k-1}, y_{k-1}\right)$, pick a component of $G\left(x_{k}, y_{k}\right)$ below but so close to $\left(x_{k-1}, y_{k-1}\right)$ that

$$
x_{k-1}-x_{k}<c\left(y_{k-1}-x_{k-1}\right), \quad \text { where } c:=b^{-1} \text {. }
$$

It is geometrically evident that

$$
y_{k-1}-x_{k-1}<x_{k-2}-x_{k-1} \text { for } k=3,4, \ldots \text {. }
$$

Then $\left\{x_{k}\right\}$ descends monotonically to a finite limit point. To see this, note that inequalities (5) and (6) imply $x_{k-1}-x_{k}<c\left(x_{k-2}-x_{k-1}\right)$, whence

$$
x_{k-1}-x_{k}<c\left(x_{k-2}-x_{k-1}\right)<\cdots<c^{k-2}\left(x_{1}-x_{2}\right)
$$

so that (let $\left.x_{0}=0\right) x_{n}=\sum_{i=1}^{n} x_{i}-x_{i-1}>x_{1}-\sum_{i=0}^{n-2} c^{i}\left(x_{1}-x_{2}\right)>x_{1}-$ $\frac{1}{1-c}\left(x_{1}-x_{2}\right)$ for all $n$. Call the limit point 0 and note that the $x_{n}$ 's are in $E$ and decrease to 0 . So by the $\limsup$ characterization of $E, 0$ is a point of right discontinuity. Find the $\eta_{0}$ associated with relation (4), and pick $k$ so large that $y_{k}<\eta_{0}$. Applying first (5) and then (6) $j$ times followed by a last application of (5) produces $x_{k+j}-x_{k+j+1}<c^{j+1}\left(y_{k}-x_{k}\right)$ for $j=0,1, \ldots$; so taking $\lim x_{n}=0$ into account we have

$$
\begin{aligned}
x_{k} & =\sum_{i \geq k}\left(x_{i}-x_{i+1}\right)<\sum_{i \geq 1} c^{i}\left(y_{k}-x_{k}\right) \\
& =\frac{c}{1-c}\left(y_{k}-x_{k}\right)=\frac{b^{-1}}{1-b^{-1}}\left(y_{k}-x_{k}\right)=\frac{1}{b-1}\left(y_{k}-x_{k}\right) .
\end{aligned}
$$

Thus $b x_{k}<y_{k}$. But this means that there is an $\eta$ just slightly larger than $x_{k}$ with $b \eta<y_{k}$. Then $[\eta, b \eta] \subset\left(x_{k}, y_{k}\right) \subset G$, so $f$ is continuous and hence 
bounded on $[\eta, b \eta]$, contrary to $\sup _{h \in[\eta, b \eta]}|f(h)-f(0)|=\infty$. This proves (C) and completes the proof of Theorem 1.

Remark. Theorem 1 is concerned with three-point schemes. The smallest possible schemes for continuity are two-point of the form

$$
f\left(x+\beta_{1} h\right)-f\left(x+\beta_{2} h\right), \quad \text { where }\left|\beta_{1}\right| \geq\left|\beta_{2}\right| \text { and } \beta_{1} \neq \beta_{2} .
$$

These are of no interest for characterization because the example of the characteristic function of a single point shows that to characterize continuity in the sense of Theorem 1 we must have $\beta_{2}=0$, and then the change of scale $h \rightarrow \frac{h}{\beta_{1}}$ shows that the condition analogous to $(3)$ is itself the definition of continuity.

Theorem 4. Assume that $f: \mathbb{R} \rightarrow \mathbb{R}$ is measurable and that for every $x$

$$
\lim _{h \rightarrow 0} \sum_{i=0}^{n} a_{i} f\left(x+b^{i} h\right)-\left(\sum_{i=0}^{n} a_{i}\right) f(x)=0,
$$

with $n \geq 2$ and $b \notin\{0,1,-1\}$. Then if $|b|>1$, relation (7) is equivalent to continuity when all of the (possibly complex) roots of the characteristic polynomial $\left(:=\sum_{i=0}^{n} a_{i} x^{i}\right)$ have moduli $>1$. If at least one root has modulus $\leq 1$, then there is a continuous on $\mathbb{R} \backslash\{0\}$, discontinuous at 0 , function satisfying (7). The situation is analogous if $0<|b|<1$, but the characteristic polynomial is then $\sum_{i=0}^{n} a_{n-i} x^{i}$.

Remarks. Special differences of this form were studied in [MZ] and [As2]. The excluded values of $b, 0,1$, and -1 correspond to cases where $\#\left\{b^{i}\right\} \leq 2$ and so are covered by either Theorem 1 or the previous remark. It is interesting that the characteristic polynomial depends on the $\left\{a_{i}\right\}$ but not on $b$.

Proof of Theorem 4. If $0<|b|<1$, set $h=k b^{-n}$ and $\beta=b^{-1}$. Then (7) becomes $\lim _{k \rightarrow 0} \sum_{i=0}^{n} a_{n-i} f\left(x+\beta^{i} k\right)-\sum a_{n-i} f(x)$ where $|\beta|>1$. Thus it suffices to treat the case where $|b|>1$.

Assume henceforth that $|b|>1$. Ease notation by writing $\phi(h)=\phi_{x, f}(h):=$ $f(x+h)-f(x)$. Then we may rewrite (7) as

$$
\lim _{h \rightarrow 0} \sum_{i=0}^{n} a_{i} \phi\left(b^{i} h\right)=0
$$

while continuity at $x$, when it occurs, has the formulation $\lim _{h \rightarrow 0} \phi(h)=0$.

Let $a$ be a root of $\sum a_{i} x^{i}$ so that $\sum_{i=0}^{n} a_{i} x^{i}=(x-a) \sum_{i=0}^{n-1} a_{i}^{\prime} x^{i}$. Then we have the following functional identities:

$$
\sum_{i=0}^{n} a_{i} \phi\left(b^{i} h\right)=\sum_{i=0}^{n-1} a_{i}^{\prime}\left(\phi\left(b^{i+1} h\right)-a \phi\left(b^{i} h\right)\right)
$$

and

$$
\sum_{i=0}^{n} a_{i} \phi\left(b^{i} h\right)=\sum_{i=0}^{n-1} a_{i}^{\prime} \phi\left(b^{i} b h\right)-a \sum_{i=0}^{n-1} a_{i}^{\prime} \phi\left(b^{i} h\right) .
$$

Note that while the $\left\{a_{i}\right\}$ are real, some $\left\{a, a_{i}^{\prime}\right\}$ may be complex.

First suppose that $|a| \leq 1$. Recall the counterexample function $F(x)=$ $|x|^{\log _{|b|}(-a)}$ given above in the proof of Theorem 1. Since $F$ satisfies (2), by 
virtue of identity (9), $F$ also satisfies relation (8). Finally since the $\left\{a_{i}\right\}$ are all real, the real part of $F$ provides a real-valued discontinuous at 0 function satisfying (8).

Now assume that all roots of the characteristic polynomial have moduli $>1$, and let $a$ be a root of that polynomial. It suffices to show that

$$
\lim _{h \rightarrow 0} \sum a_{i}^{\prime} \phi\left(b^{i} h\right)=0 \quad \text { everywhere, }
$$

for repeating the argument $n-1$ times will lead to

$$
\lim _{h \rightarrow 0} \sum_{i=0}^{0} a_{i}^{(n)} \phi\left(b^{i} h\right)=0 \text { for all } x
$$

(note $a_{n}=a_{n-1}^{\prime}=a_{n-2}^{\prime \prime}=\cdots=a_{0}^{(n)}$ ), i.e., that $f$ is continuous at every $x$.

The proof is very much like the positive part of the proof of Theorem 1, since relations (3) and (10) are almost identical. The reduction there to $b>$ 1 works here also since $b$ is real, but, since $a$ may be complex, $a^{2}$ is not necessarily positive so that we may not assume that $a$ is positive. From the original assumption (7) we have by Theorem 2 that $f$ is continuous a.e. So letting $E:=\left\{x: \lim _{h \rightarrow 0} \sum a_{i}^{\prime} \phi\left(b^{i} h\right) \neq 0\right\}$ we see that $|E|=0$. The proof is completed by carefully tracking the proofs of steps (B) and (C), replacing $a$ by $|a|$ as required.

Theorem 5. Suppose that $p_{0}=0$ and, for $i \in\{1,2, \ldots, n\}, p_{i}$ satisfies $\Re p_{i} \geq$ 0 . If the characteristic quasipolynomial $\sum_{i=0}^{n} a_{i} z^{p_{i}}$ has a root of modulus $\leq$ 1 , then for any $b$ with $|b|>1$, there is a function $f: \mathbb{R} \rightarrow \mathbb{R}$ which is discontinuous at 0 and continuous elsewhere with the property that for every $x \in \mathbb{R}$

$$
\lim _{h \rightarrow 0} \sum_{i=0}^{n} a_{i} f\left(x+b^{p_{i}} h\right)-\left(\sum_{i=0}^{n} a_{i}\right) f(x)=0 .
$$

Proof. The counterexample function is the same as the one given in the proof of Theorem 4, namely the real part of $|x|^{\log _{|b|}(-a)}$, where $a$ is a root of the characteristic quasipolynomial satisfying $|a| \leq 1$.

Theorem 6. Let $0<b_{0}<b_{1}<\cdots<b_{n}$, and suppose that $\left|a_{0}\right|>\sum_{i=1}^{n}\left|a_{i}\right|$. If for every $x \in \mathbb{R}$

$$
\lim _{h \rightarrow 0} \sum_{i=0}^{n} a_{i} f\left(x+b_{i} h\right)-\left(\sum_{i=0}^{n} a_{i}\right) f(x)=0,
$$

then $f$ must be continuous.

Proof. Use Lemma 1. Steps (A), (B), and (C) follow without any change.

To further support the conjecture, we note that (with the aid of Theorem 6), Theorem 4 can be extended to the case where the characteristic quasi polynomial is a product of polynomials of single variables. We omit the details.

Theorem 7. Suppose that $a_{0} a_{n} \neq 0$ and $p_{0}<\Re p_{1} \leq \cdots \leq \Re p_{n-1}<\Re p_{n}$. Then the quasipolynomial $P(z):=\sum_{i=0}^{n} a_{i} z^{p_{i}}$ has at most a finite number of roots on any sheet of its Riemann surface. 
Proof. Fix a sheet by defining $z^{p}:=e^{p(\ln |z|+i \arg z)}$, where $\arg z$ is constrained to lie in a fixed half-open interval of length $2 \pi$. This is necessary, for otherwise a quasipolynomial such as $x^{\pi}-1$ would have infinitely many roots. To fix ideas, say that $-\pi<\arg z \leq \pi$. Since $\left|z^{p}\right|=|z|^{\Re p} e^{-\Im p \arg z}$ and $|\arg z| \leq \pi$, it is easy to see that the first term of $P$ is dominant for $|z|$ small. Similarly, the last term of $P$ is dominant for $|z|$ large. Thus the only possible zeros of $P$ lie in an annulus $A$ centered at $z=0$, but $P$ is analytic on its associated Riemann surface $S:=\{z: 0<|z|<\infty,-\infty<\arg z<\infty\}$. Hence its zeros have no accumulation points on $A$ since $A \subset S$. Thus $P$ can have at most finitely many zeros.

To see that both of the strict inequalities appearing in the statement of Theorem 7 are necessary, notice that $x^{i}-1=0$ is satisfied by $e^{2 \pi n}$ for every integer $n$.

Theorem 8. Suppose that $d \geq 1$ and

$$
\sum_{i=0}^{n} a_{i} b_{i}^{j} \begin{cases}=0, & j=0,1, \ldots, d-1, \\ \neq 0, & j=d .\end{cases}
$$

Let $f$ be a Lebesgue measurable function satisfying the following difference equation:

$$
\sum_{i=0}^{n} a_{i} f\left(x+b_{i} h\right)=0 \quad \text { for all real } x \text { and all real } h .
$$

Then $f$ is a polynomial of degree at most $d-1$.

Proof. We may assume $a_{0} \neq 0$. Divide equation (11) by $a_{0}$, replace $x$ by $x-b_{0} h$, set $\alpha_{i}:=-a_{i} / a_{0}$ and $\beta_{i}:=b_{i}-b_{0}$ for $i=1, \ldots, n$, and transpose all but the first term to get

$$
f(x)=\sum_{i=1}^{n} \alpha_{i} f\left(x+\beta_{i} h\right) \text { for all real } x \text { and all real } h .
$$

First note that $f$ is locally bounded; for if not, then there is a convergent sequence $\left\{x_{k}\right\}$ so that $\left|f\left(x_{k}\right)\right| \rightarrow \infty$. Let $M$ be a large integer. If $\mathbf{k}$ is sufficiently large, $\left|f\left(x_{k}\right)\right| \geq n \max \left\{\left|\alpha_{i}\right|\right\} M$. From equation (12), it follows that for every choice of $h$ there is a $j \in[1, n]$ so that $\left|f\left(x_{k}+\beta_{j} h\right)\right| \geq M$. Thus if, for $j=1, \ldots, n, E_{j}:=\left\{h \in[0,1]:\left|f\left(x_{k}+\beta_{j} h\right)\right| \geq M\right\}$, then $[0,1]=\bigcup E_{j}$. Consequently there is a $j_{o}$ so that

$$
\left|E_{j_{o}}\right| \geq \frac{1}{n}
$$

Let $I:=\left[\inf \left\{x_{k}\right\}+\min _{j}\left\{\beta_{j}, 0\right\}, \sup \left\{x_{k}\right\}+\max _{j}\left\{\beta_{j}, 0\right\}\right]$ and $F_{M}:=\{t \in$ $I:|f(t)| \geq M\}$. Let $c:=\min \left\{\left|\beta_{j}\right|\right\} \frac{1}{n}$. From equation (13), we see that $\left|F_{M}\right| \geq\left|\beta_{j_{o}}\right|\left|E_{j_{o}}\right| \geq c$. But the sets $\left\{F_{M}\right\}_{M=1}^{\infty}$ are nested, so their intersection must have measure at least $c$ and $|f|$ exceeds every $M$ on that intersection, which contradicts the fact that Lebesgue measurable functions are finite almost everywhere. 
Since $f$ is locally bounded, we may form its indefinite integral

$$
F(x):=\int_{0}^{x} f(t) d t
$$

Integrate equation (12) from 0 to 1 in the variable $h$ to get

$$
f(x)=\sum_{i=1}^{n} \frac{\alpha_{i}}{\beta_{i}}\left(F\left(x+\beta_{i}\right)-F(x)\right) .
$$

Equations (14) and (15) bootstrap $f$ into the class $C^{\infty}$. Since $f$ is locally bounded, by (14) $F$ is continuous; then by (15) $f$ is continuous; then by (14) $F^{\prime}$ is continuous; then by (15) $f^{\prime}$ is continuous; then by (14) $F^{\prime \prime}$ is continuous; etc.

Finally differentiate equation (11), with respect to $h, d$ times. Then set $h=$ 0 and divide by $\sum_{i=0}^{n} a_{i} b_{i}^{d}$ to get that $f$ has identically zero $d$ th derivative, which is to say that it is a polynomial of degree at most $d-1$.

Remarks. Note that Theorem 8 even holds when $d=0$, provided we interpret the set of all polynomials of degree -1 to be the singleton consisting of the function 0 . Also $d \leq n$ in Theorem 8 , since $\sum a_{i} b_{i}^{j}=0, j=0, \ldots, n$, would be a homogeneous system and $\operatorname{det}\left(b_{i}^{j}\right) \neq 0$. If one looks for a hypothesis between that of Theorem 1 and Theorem 8 , one might try to replace "for all $x$ and for all $h$ " by "for all $x$ and for all $h$ with $|h|<H(x)$, where $H(x)>0$ for every $x$ ". This concept, which might be called locally identically zero, is quite different from the hypothesis of Theorem 8. To see this, consider the function $s(x):=\operatorname{sgn}(x)$ which satisfies $s(0+h)-2 s(0)+s(0-h)=0$ for all $h$, while, for $x \neq 0, s(x+h)-2 s(x)+s(x-h)=0$ provided $|h|<|x|$. We will not pursue the idea of locally identically zero any further here.

\section{ACKNOWLEDGMENT}

We thank the referee for a careful reading and helpful suggestions.

\section{REFERENCES}

[As1] J. M. Ash, Generalizations of the Riemann derivative, Trans. Amer. Math. Soc. 126 (1967), 181-199.

[As2] $\longrightarrow$ A characterization of the Peano derivative, Trans. Amer. Math. Soc. 149 (1970), 489-501.

[MZ] J. Marcinkiewicz and A. Zygmund, On the differentiability of functions and summability of trigonometric series, Fund. Math. 26 (1936), 1-43.

[Zy] A. Zygmund, Trigonometric series, Vol. 1, 2nd rev. ed., Cambridge Univ. Press, New York, 1959.

Department of Mathematics, DePaul University, Chicago, Illinois 60614

Department of Mathematics, California State University, San Bernardino, CaliforNIA 92407 